\title{
De la donnée à l'information : de l'importance des conventions dans les organisations
}

\author{
From Data to Information: about the importance of conventions in
} organisations

\author{
Gabriel Colletis ${ }^{1}$, Etienne Fieux ${ }^{2}$, Anne Isla $^{3}$, Adrien Peneranda ${ }^{4}$ \\ ${ }^{1}$ LEREPS, Université Toulouse 1, Gabriel.Colletis@ut-capitole.fr \\ ${ }^{2}$ IMT, Université Toulouse 2, Etienne.Fieux@math.univ-toulouse.fr \\ ${ }^{3}$ LEREPS, Université Toulouse 2, Anne.Isla@univ-tlse2.fr \\ ${ }^{4}$ LEREPS, Sciences Po Toulouse, Adrien.Peneranda@sciencespo-toulouse.fr
}

\begin{abstract}
RESUME. Nous évoluons tous en permanence dans de multiples organisations et sommes assaillis par un flux ininterrompu de données et d'informations, flux que nous contribuons à alimenter. Ces données et informations, distinguées dans le texte, ne sont ni neutres ni objectives mais sont le résultat d'opérations diverses générées par les organisations qui forment ou utilisent lesdites « données ". Ces opérations obéissent à quatre types de conventions : des conventions de quantification, des conventions de contextualisation, des conventions d'énonciation, des conventions sémiotiques, enfin. Reflet de la doxa, les conventions en sont aussi le produit et façonnent les normes qui encadrent la vie économique et le fonctionnement des organisations. Deux exemples de travail des conventions dans une organisation sont proposés dans le texte : celui d'un hôpital et du tableau de bord de celui-ci supposé refléter sa performance ; celui du partage de la valeur ajoutée. En même temps cadre et résultat de l'action, instituantes et instituées comme l'ont montré les "conventionnalistes", les conventions et la dispute autour de celles-ci constituent le reflet de la vie de la démocratie dans les organisations. La question de l'élaboration des conventions est trop souvent ignorée et son importance sous-estimée par les acteurs potentiels de la dispute dans les organisations et, singulièrement, les syndicats. ABSTRACT. We are constantly evolving in multiple organizations and we have to deal with an uninterrupted flow of data and information, which we contribute to feed. These data and information, clearly differentiated in this paper, are neither neutral nor objective but are the result of various operations generated by the organizations that form or use the so-called "data". These operations obey four types of conventions: quantification conventions, contextualization conventions, stating conventions, semiotic conventions, finally. Conventions reflect the doxa and are shaping the norms that frame the economic life and the functioning of organizations. The example of a hospital illustrates these conventions in the article. Another example is used: the added value divide. The balance scorecard of this hospital reflects his performance. The conventions are simultaneously process and result of the action, instituting and instituted, as the "conventionalists" have shown. The conventions and the dispute around them testify to the life of democracy in organizations. The question of the elaboration of conventions is too often ignored and its importance underestimated by the potential actors of the dispute in the organizations and, in particular, the unions.
\end{abstract}

MOTS-CLES. données, informations, systèmes d'information, organisations, conventions, quantification, contextualisation, tableau de bord, démocratie.

KEYWORDS. data, information, information systems, organisations, conventions, balance scorecard, quantification, contextualization, democracy.

\section{Introduction : les organisations et les données sont partout}

Les organisations se sont étendues dans tous les domaines de la vie sociale : de la petite entreprise familiale jusque dans les écoles, les hôpitaux et bien évidemment au cœur des administrations publiques comme à la tête de l'État. "L'homme moderne ne peut agir qu'à travers et au sein de grandes organisations » [MAR 64]. Or il n'y a pas d'organisation sans systèmes d'information et il n'y a pas de systèmes d'information sans organisation. Le but des organisations complexes étant de s'organiser pour décider [SIM 80], ces systèmes sont principalement orientés vers le traitement de l'information. L'organisation contemporaine a ainsi la particularité d'être inséparable de son système d'information [LEM 86]. Les systèmes d'information ne laissent souvent aucune latitude aux employés qui doivent se soumettre et s'adapter avec «docilité » [SIM 83] à leur logique d'autant que leur 
pouvoir normatif est très puissant et souvent masqué. Ce processus se déroule en effet par la mise en œuvre de technologies plus ou moins visibles comme l'avait déjà pointé Michel Berry [BER 83].

Dans ce contexte, le principal problème rencontré par les individus dans leur processus d'adaptation à ces nouveaux environnements est celui de la surabondance informationnelle. Ce trait caractéristique de notre «société de l'information » provoque une surcharge tant au niveau du potentiel d'attention des utilisateurs de systèmes d'information qu'au niveau de leurs possibilités de traitement de l'information. La productivité des individus n'est pas en cause ici car il s'agit de deux limitations humaines fortes : la capacité d'attention (c'est-à-dire l'allocation des ressources cognitives) et la capacité cognitive de traitement de l'information qui l'une comme l'autre ne sont en rien "parfaites » ainsi que l'a montré Herbert Simon avec ses travaux sur la rationalité limitée [SIM 91]. L’information produite par les machines et les hommes ces dernières années est jugée supérieure à toute celle produite depuis l'origine de l'humanité et crô̂t à un rythme exponentiel [SAU 14] [LYM 03]. Cependant, les connaissances ne croissent pas au même rythme que les informations et la richesse des connaissances mobilisables peut même décroître dans certaines organisations, notamment lorsque l'absence de distinction claire entre données et informations désoriente l'action des individus. Cette absence de distinction anéantit aussi en grande partie le caractère démocratique de l'organisation.

Données et informations sont régulièrement confondues et présentées dans les médias comme objectives et neutres, à la base de vérités absolues. Or, les données ne sont pas données. C'est le sujet qui crée les données. Au Canada, une image familière dit que les données « ne poussent pas aux arbres » (on ne cueille pas les données !). Les anglo-saxons ont l'avantage de disposer du terme « data » pour désigner les «données » et «to give» pour le verbe «donner». «The data are not given » est une évidence dans la langue de Shakespeare. Une donnée est un «signe » auquel on prête attention et qui, lorsqu'il est enregistré, l'est le plus souvent dans un code convenu [MEL 79]. L'information est construite à partir d'un ensemble de données que l'on structure et que l'on situe et qui produit du sens. Selon la célèbre définition de Bateson [BAT 79] : «Une unité d'information (bit of information) peut se définir comme une différence qui produit une autre différence », c'est-à-dire un message qui modifie la façon qu'a le destinataire de percevoir quelque chose. Ainsi, l'information est souvent préalable à une décision ou une action. La donnée a un potentiel sémantique. C'est-à-dire que si elle est là, si elle a une forme que l'on peut reconnaître, alors on peut faire l'hypothèse qu'elle est susceptible d'avoir un ou plusieurs sens. L'information « conjoint inséparablement trois composantes : la forme physique (dit signe ou composante syntaxique), les significations dont ce signe peut être porteur (la composante sémantique), et les actions contextuelles suscitées par la réception de ce signe susceptible de prendre des significations différentes - et parfois imprévues par l'émetteur - selon les contextes de réception » [LEM 95]. Cette perspective rejoint de nombreuses recherches qui définissent les systèmes d'information (SI) comme les interfaces « d'un projet et d'un contexte » [LEM 06], c'est-à-dire des « espaces de rencontres entre autant de projets et de contextes » [ROW 06]. «Un SI se présente donc comme un système de formalisation de représentations qui rend ces formalisations opérationnelles, et accessibles (et agissantes), au travers de codifications particulières » [SAL 15]. De plus, si les individus peuvent constituer ensemble des règles de partage de l'information, l'interface ainsi conçue peut en retour guider leur comportement. Les acteurs décident des informations qu'ils vont aller construire, chercher, et créer, traiter eux-mêmes, dans l'espoir que ces informations qu'ils ont eu l'idée d'aller chercher les aident en effet à décider de l'action qu'ils vont entreprendre. On n'informe donc pas la décision, on décide de l'information.

«Les données ne sont pas données ». Cette proposition, formulée de différentes façons (dont [SAL 10]), tend à être reconnue par un nombre croissant de chercheurs si ce n'est dans la vie courante ou le débat public. À travers des formulations diverses, les chercheurs qui défendent cette proposition, que l'on peut considérer comme «constructiviste », suggèrent que les données ne sont pas neutres ou objectives mais sont le résultat d'opérations diverses générées par les organisations qui forment ou utilisent lesdites « données ». 
La première partie de l'article présente les notions de convention et d'organisation sur lesquelles s'appuie notre raisonnement à partir de travaux pluridisciplinaires en économie et en sciences de gestion. Nous adoptons en particulier une perspective d'ingénierie pour montrer l'importance des systèmes d'information (SI) comme dispositifs de pouvoir au sein des organisations. La deuxième partie de l'article propose une nouvelle typologie des conventions afin de mieux saisir la manière par laquelle s'opère le passage des données aux informations. Des exemples concrets illustrent chacune de ces conventions, en particulier les modalités de calcul du PIB et le partage de la valeur ajoutée dans les entreprises. Nous mobilisons enfin les nouvelles conventions que nous avons mises en évidence dans le cadre d'une étude de cas consacrée à la démarche de pilotage d'un hôpital au moyen d'un tableau de bord. Cet outil est un système d'aide à la décision (SAD) qui utilise des indicateurs issus du SI pour fournir certaines représentations de données sélectionnées aux décideurs de l'organisation. Notre analyse montre que ces constructions sont basées sur des conventions qui ne font généralement pas l'objet d'une dispute démocratique dans les organisations. Nous proposons en conséquence plusieurs pistes, faisant notamment appel à l'expertise syndicale, pour revivifier la démocratie en entreprise autour de ces questions qui ne peuvent être réduites à leur simple dimension technique.

\section{Conventions et organisation}

Avant d'approfondir ce que nous entendons dans cet article par ces opérations qui forment ou utilisent les «données », nous voudrions resituer notre propos dans la perspective de SI compris comme « appareillage de la doxa ».

Notre hypothèse centrale se résume de la manière suivante : la vie de la démocratie dans les organisations dépend de la façon dont fonctionnent les SI et, plus spécifiquement, au sein de ceux-ci, de la manière dont se construisent les conventions. Afin de situer notre propos, nous indiquerons que nous reprenons à notre compte dans ce texte, sans les développer plus avant, les (cinq) composantes de la démocratie dans les organisations telles que proposées dans la contribution de Salles, Bour et Jardat dans le présent numéro [SAL 20] : champs de l'autonomie et types de décisions concernées, statut de citoyen et de non-citoyen dans l'organisation, lien entre capacité à participer à la prise de décision et information de l'acteur concerné, organisation de la confrontation d'opinions différentes, modes de gouvernement des organisations et leur équilibre.

$\mathrm{Si}$ nous considérons que les SI et les conventions qui les sous-tendent sont des composantes essentielles de l'appareillage de la doxa, c'est que nous reprenons à notre compte la construction proposée par Salles et Colletis [SAL 13]. La perspective retenue par les auteurs est celle d'une ingénierie pour dé/construire des dispositifs de pouvoir. Cette ingénierie s'appuie sur un modèle qui distingue et explicite trois niveaux dans les dispositifs de pouvoir. Le premier niveau est celui des normes qui encadrent concrètement la vie économique. Le deuxième niveau est celui des principes qui orientent les normes, qui établissent leur cadre conceptuel et méthodologique. Le troisième niveau renvoie aux visions du monde sous-jacentes aux principes et aux normes : les doxai, dont font partie les missions et la finalité d'une organisation (ou la représentation que l'on en a).

Les conventions auxquelles nous nous intéressons ici s'inscrivent au sein du niveau des principes. Ce niveau, selon Salles et Colletis [SAL 13], comprend, en effet, entre autres :

- le langage de l'organisation ou celui employé au niveau « macro »: termes employés, langue d'expression choisie, etc. ainsi que les axes de communication privilégiés ;

- les conventions (explicites ou implicites). Comme, par exemple, les conventions salariales, les conventions de (taux) de change, mais aussi les conventions implicites s'agissant des « attentes » des marchés financiers...;

- les principes et catégories du droit national, européen et international ; 
- les structures des systèmes d'information : conventions d'équivalence pour quantifier une « réalité », principes de construction de nomenclatures, des jeux de critères comparatifs, etc.

À ce stade du développement il est nécessaire de faire le point sur ce que l'on entend par convention et organisation [ISL 02] [ISL 05]. Notre position consiste à affirmer fortement que seules des conventions faisant l'objet d'une dispute ${ }^{1}$ et d'un consensus sont de nature à rendre un SI démocratique. Or force est de constater que cette dispute et ce consensus (sous la forme d'un compromis) sont des exceptions. Les conventions sont le plus souvent imposées, ne faisant pas l'objet de débats au sein de l'organisation.

L'organisation peut être définie comme un ensemble de dispositifs cognitifs collectifs permettant au groupe, par voie d'apprentissage collectif, d'atteindre des objectifs globaux [FAV 89]. L'organisation est constituée des acteurs et de leurs actions, elle sera qualifiée, selon les auteurs, d'organisation active [LEM 90], d'unité active [PER 69], d'organis-action [MOR 77]. L'organisation traduit les interrelations, les articulations et la structure du système. Elle permet d'encadrer l'action des acteurs et peut libérer l'action individuelle sous certains aspects. L'organisation est une structuration d'espaces de négociation et de jeux entre acteurs [FRI 92].

Dans la décomposition des dispositifs de pouvoir en trois niveaux : normes, principes et doxa, nous avons vu que les conventions se situent au niveau des principes. Les conventions peuvent donc être abordées comme le nœud entre les normes qui encadrent la vie économique (règles constitutives qui déterminent les procédures d'élaboration des règles et la place des acteurs) et les règles de conduite qui définissent directement le comportement des acteurs [ISL 02] [ISL 05].

Étymologiquement, une convention est ce qui résulte d'un accord réciproque par opposition aux lois naturelles. Le mot vient du latin conventio qui signifie pacte, assemblée, accord. Cet accord, le résultat de ce processus de prise de décision-résolution organisationnelle [LEM 90] est en constante construction et résulte d'un phénomène de rétroaction. Ainsi, on entend convention dans le sens de celui élaboré par les économistes des conventions : les conventions sont, en même temps, cadre et résultat de l'action, cadre contraignant les sujets et résultat d'actions individuelles, instituantes et instituées. La convention «désigne le dispositif constituant un accord de volonté tout comme son produit doté d'une force normative obligatoire » [DUP 89].

Décomposer les dispositifs de pouvoir, les processus de prise de décision au sein de l'organisation, et regarder comment se construisent les règles qui coordonnent les comportements, les conventions sous-jacentes et la doxa que cela exprime, nous permet de montrer qu'il n'existe aucune neutralité, naturalité, inéluctabilité ou objectivité d'un SI. Il y a un choix. Les règles instrumentalisent une vision du monde. Les comportements individuels sont inscrits dans des représentations collectives qui ellesmêmes sont construites par les acteurs individuels : en même temps cadre et résultat de l'action. D'autre part, cette décomposition nous permet de mettre à jour le caractère démocratique ou non d'un SI.

Derrière toute règle, derrière tout indicateur, il $\mathrm{y}$ a des conventions plus ou moins implicites. Mettre en lumière ces conventions permet de questionner les aspects démocratiques, la construction de conventions légitimes. Un des enjeux et de redonner à chacun les moyens, la connaissance, les capacités à former un jugement et à participer aux prises de décisions ${ }^{2}$.

\footnotetext{
${ }^{1}$ Le terme « dispute » est employé ici dans son sens étymologique qui renvoie au verbe latin putare qui signifie «penser ». Comme le souligne Nodé-Langlois, la dispute consiste à rechercher ce qu'il faut penser, sur une question donnée, par le moyen d'une confrontation qui permet d'aboutir à une conclusion vraie alors que la simple discussion peut être « un affrontement sans issue de positions opposées » [NOD 11].
}

\footnotetext{
${ }^{2}$ Le fil conducteur de la démarche conventionnaliste « est donc la question de la coordination et du pouvoir dans les interactions économiques et sociales. Les problèmes posés concernent leur mise en œuvre démocratique et, symétriquement, la solution des conflits (conflits d'intérêt, mais aussi conflits de principes fondateurs). Par rapport à d'autres courants de recherche, nous visons une 


\section{Quatre types de conventions}

Les conventions ne se confondent pas avec les missions de l'organisation, elles en sont la traduction. Nous considérerons dans cette contribution quatre types de conventions: les conventions de quantification, celles de contextualisation, celles d'énonciation, celles, enfin, que nous désignons comme des conventions sémiotiques.

Les quatre conventions que nous nous proposons de retenir et d'analyser dans ce texte sont complémentaires les unes des autres. Elles s'articulent et s'agencent pour passer de la donnée à l'information, voire pour produire directement de l'information. Elles sont complémentaires et ce qui les différencie les unes des autres n'est pas toujours simple à distinguer.

Sans qu'il s'agisse dans ce texte de produire une analyse théorique des conventions, il est sans doute utile de rappeler que ce que l'on peut désigner comme «l'économie des conventions » est pluriel et, sous certains aspects, hétérogène. La diversité des approches de l'économie des conventions peut être appréhendée de différentes manières : cognitiviste ou pragmatiste, centrée sur la réalisation du produit ou encore mettant plutôt l'accent sur les mécanismes de coordination ${ }^{3}$. L'usage présent de l'économie des conventions se rattache plutôt aux travaux de type cognitiviste et met l'accent sur les questions de coordination.

Nous montrons à présent qu'il est possible de mobiliser ces quatre types de convention pour révéler le passage de la donnée à l'information.

\subsection{Les conventions de quantification}

Les conventions de quantification ont été clairement mises en évidence par Desrosières qui affirme que «La quantification ne reflète pas la réalité, mais en revanche contribue à la façonner, à la transformer, sinon à la créer » [DES 03]. L'auteur rappelle que "Le verbe quantifier, dans sa forme active (faire du nombre), suppose que soit élaborée et explicitée une série de conventions d'équivalences préalables, impliquant des comparaisons, des négociations, des compromis, des traductions, des inscriptions, des codages, des procédures codifiées et réplicables, et des calculs conduisant à la mise en nombre. La mesure proprement dite vient ensuite, comme mise en œuvre réglée de ces conventions » [DES 10].

Les données et informations qui font partie de notre quotidien, comme de celui des organisations, se présentent sous une forme objectivée alors qu'elles reposent sur des conventions construites, lesquelles résultent de choix.

À titre d'exemple, le périmètre du Produit Intérieur Brut (PIB), qui est supposé mesurer la richesse produite par une nation en un an, et qui sert, par exemple, de référence pour évaluer l'endettement d'un État, suggère de se demander quelles activités entrent ou n'entrent pas dans son calcul :

- Le travail d'un enseignant? (oui)

- La prostitution? (non $\left.{ }^{4}\right)$

intégration originale entre démocratie et économie. Au lieu de localiser la démocratie dans un espace politique spécialisé ou dans l'espace de la protection sociale, nous inscrivons l'exigence de démocratie dans les actions économiques elles-mêmes » [EYM 06].

${ }^{3}$ Pour une synthèse succincte des différents clivages qui traversent l'économie des conventions, on pourra se reporter à [KIR 02].

\footnotetext{
${ }^{4}$ Non pour la France mais oui en Irlande, aux Pays-Bas, en Espagne, en Italie, au Royaume-Uni, ce qui montre que les conventions ne sont pas les mêmes d'un pays à l'autre. « À la demande d'Eurostat, plusieurs pays (Espagne, Royaume-Uni, Italie...) ont décidé d'intégrer de nouvelles normes de calcul du PIB. À chaque fois, cela s'est traduit par une révision à la hausse de leur produit intérieur brut. Même si elle a le souci d'aligner les statistiques de la France sur celles de ses voisins européens, l'Insee s'est refusé à intégrer la prostitution (de "réseaux"), estimant qu'il ne s'agissait probablement pas de "transactions commerciales consenties librement" ». https://www.latribune.fr/economie/france/le-trafic-de-drogue-mais-pas-la-prostitution-bientot-integre-dans-le-calcul-du-pib-francais- 
- Les activités domestiques ? (non)

- L'activité des firmes « étrangères » en France ? (oui)

-L'activité des firmes françaises à l'étranger? (non, mais oui pour le PNB)

-Le commerce de la drogue? (oui depuis 2018).

Observons à présent les conventions de quantification à l'intérieur d'une entreprise : nous pouvons identifier que les salaires sont conventionnellement calculés comme des charges qu'il faut réduire parce qu'ils viennent en diminution du résultat de l'entreprise (et donc de la part des bénéfices susceptibles d'être attribués aux détenteurs du capital). Nous expliciterons cet exemple de quantification dans l'encadré suivant.

Nous voudrions insister sur deux caractéristiques des conventions de quantification :

- celles-ci sont souvent mal connues,

- leur élaboration peut faire l'objet d'une dispute, d'un consensus...ou non.

Les conventions de quantification vont modifier les comportements des individus de l'organisation et, partant, la mission de l'organisation elle-même. Robert Salais [SAL 10] a étudié l'exemple de la police nationale dans laquelle la culture de la performance entraîne un besoin de délinquance. En effet, s'il n'y a plus de délinquance, alors il n'y a plus de performance puisqu'on ne peut pas comptabiliser les faits de délinquance évités. Si le fondement du service public assumé par la police partait d'une logique de prévention et de baisse de la délinquance, la culture du résultat et de la quantification transforme cette logique en une logique de répression: il est nécessaire que la délinquance se maintienne, voire s'amplifie afin d'obtenir des résultats. Comme le souligne Salais, « Rapporter des progrès d'une politique de prévention relève davantage d'une appréciation qualitative que quantitative. [Mais] Le «problème » qu'une telle politique [basée sur une appréciation qualitative] pose est, en outre, qu'elle interdit une stratégie de communication politique centrée sur l'affichage de résultats quantitatifs, autrement dit sur une utilisation politique de l'argument statistique » [SAL 10].

L'exemple de la santé est lui aussi symptomatique ${ }^{5}$. Dans l'évaluation de l'activité des infirmières, le temps passé avec le patient n'est pas pris en compte. Or l'hôpital est censé répondre aux besoins des patients, à leur perception des soins, etc. Les conventions de quantification modifient le statut des acteurs. L'usager de l'hôpital change de statut, il n'est plus le patient doté d'une conscience et d'une perception subjective du soin mais un simple consommateur de soin. Le policier n'est plus le chargé de mission doté d'une conscience et soucieux d'offrir un service de qualité et, en l'occurrence, une baisse de la délinquance et une société plus sereine, mais un producteur préoccupé du rapport « coûtbénéfice » et de la maximisation du score du service qu'il propose. L'usager des services publics n'est plus un citoyen inscrit dans la vie de la cité mais « un consommateur uniquement préoccupé du rapport "qualité-prix" du service ou produit qu'il achète » [SAL 10]. L'objectif n'est pas d'améliorer réellement les situations ou de poursuivre la mission de l'organisation mais d'obtenir la performance quantitative souhaitée, quelle qu'en soit la méthode. Le risque est de perdre toute référence autre que rhétorique à la mission, voire à la raison d'être de l'organisation. Policiers, patients, citoyens sont conduits à se comporter en «mercenaires» motivés par le gain ou en free riders (passagers clandestins) intéressés par la seule consommation de ressources. 


\section{L’exemple du partage de la valeur ajoutée}

Les normes de gestion reflètent une façon d'appréhender le «capital », «l'humain » et la «nature » ainsi que leurs relations. Elles comportent des conventions de quantification plus ou moins explicitées. Dans le cas de l'entreprise, si on estime que la finalité de celle-ci est de produire et de vendre des biens et des services, alors le revenu global de l'entreprise équivaut à la valeur ajoutée qu'elle génère (chiffre d'affaires moins consommations intermédiaires). La valeur ajoutée est à la fois le revenu de l'entreprise et la source des revenus de ceux qui participent à la production de ce revenu. La valeur ajoutée permet de financer les salaires des dirigeants et du personnel, de rémunérer les intérêts des banques, les impôts et les taxes demandés par l'État. La valeur ajoutée permet aussi d'assurer l'autofinancement de l'entreprise (les amortissements et les parts réinvesties du résultat) et de verser les dividendes. C'est donc la valeur ajoutée qui permet de couvrir le coût global de la structure qu'est l'entreprise (coût du travail et coût du capital).

Les normes de gestion actuelles, les mesures et quantifications qui y sont attachées, ne sont pas exemptes de conventions sous-jacentes reposant sur une représentation de la finalité de l'entreprise fort différente de celle qui vient d'être exposée. Ces conventions, aujourd'hui dominantes, ont de nombreuses conséquences.

Ainsi, les conventions mobilisées pour le partage de la valeur ajoutée reflètent la vision que l'on a de l'entreprise, de son organisation, de son rôle social et de ses finalités : outil de travail pour les salariés ou de rendement pour les actionnaires ? Production pour répondre aux besoins sociaux ou pour enrichir les détenteurs de capitaux ? Instrument pour négocier le virage écologique nécessaire ou, de facto, détruisant la planète?

Dans la comptabilité telle qu'elle est pratiquée, la valeur ajoutée est répartie en salaires et en profits (voir Figure 1). Dès ce partage, il est sous-entendu que les salaires représentent un coût alors que les profits représentent « ce qui reste à l'entreprise » comme si les salariés ne faisaient pas partie de l'entreprise 6 . De «ce qui reste à l'entreprise», c'est-à-dire le profit, sont déduits les amortissements et les remboursements d'emprunt. On en déduit alors les bénéfices bruts auxquels on retranche les impôts pour obtenir le bénéfice net. Ce bénéfice net est partagé entre les investissements, nécessaires pour la survie de l'entreprise, et les dividendes, c'est-à-dire la rémunération des actionnaires. Dans ce partage de la valeur ajoutée, la rémunération de l'actionnariat n'est pas représentée comme un coût alors que le salaire l'est.

Dans cette vision comptable de l'entreprise, le travail est un coût. Cette vision est par ailleurs clairement exprimée par les expressions « ressources humaines » ou «capital humain », comme si les humains étaient une ressource à l'instar du pétrole, des pièces détachées ou autres biens marchands. Dans un capitalisme financiarisé comme nous le connaissons depuis les années 1980, l'objectif de la grande entreprise est de faire du profit... pour les actionnaires. Cet objectif se reflète dans l'évolution du partage de la valeur ajoutée de plus en plus favorable au capital et au versement de dividendes depuis les années 1970.

\footnotetext{
${ }^{6}$ Précisons qu'en droit une « entreprise » n'existe pas. La seule entité qui existe est la société. Pour produire, la société a besoin de capital (matières premières, machines, ateliers, etc.) et de travail. La société est une personne morale et en tant que telle, elle est inappropriable. Aucun acteur économique ne peut se revendiquer propriétaire d'une entreprise, a fortiori, aucun actionnaire n'est propriétaire de l'entreprise (un actionnaire n'est propriétaire que de ses actions). 


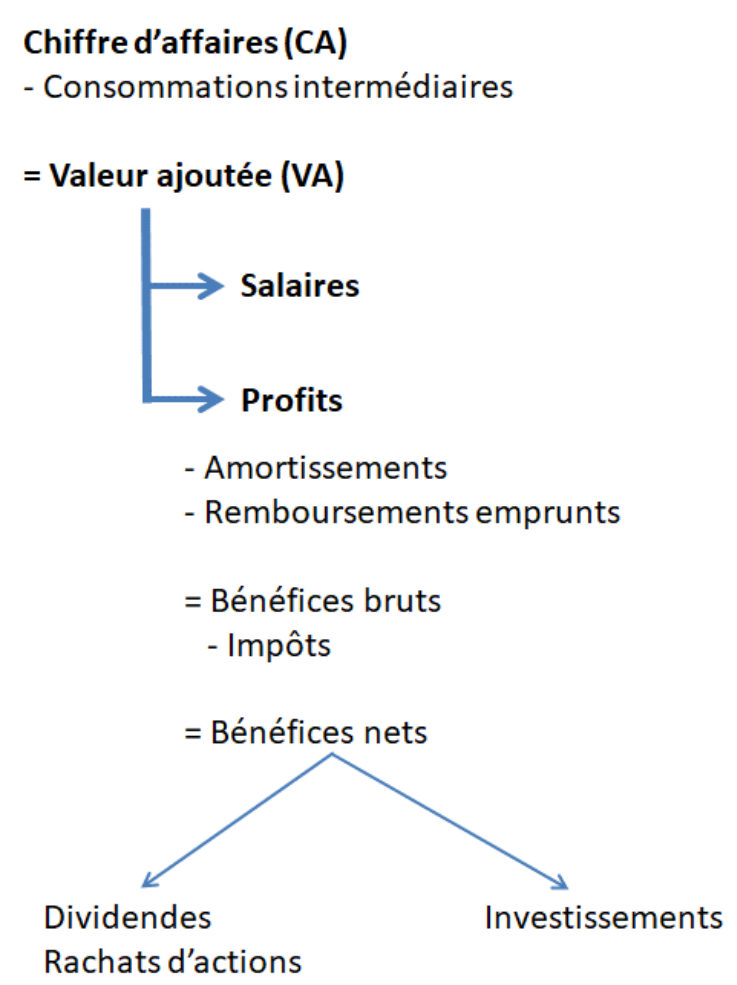

Figure 1. Le partage de la valeur ajoutée selon les normes comptables en vigueur [ISL 19]

Cette façon de concevoir le partage de la VA n'est pas unique. Elle est basée sur des conventions particulières. Elle a la particularité de considérer que le travail n'est qu'un coût et de ne pas faire figurer la reproduction ou la destruction environnementale induite par le processus productif. Les travaux d'un certain nombre de chercheurs et de praticiens ouvrent d'autres perspectives. Il est possible, comme le proposent Daniel Bachet, Jacques Richard et Alexandre Rambaud, par exemple, de traiter tous les apporteurs d'actifs de la même façon. Que ces actifs soient des apports financiers ou de travail. Il est également possible de prendre en compte le développement de l'entreprise dans son contexte écosystémique.

Dans cette perspective, certains auteurs vont apporter une vision complémentaire en proposant de tenir compte de la conservation systématique de la nature et de l'être humain. Ainsi, au lieu d'avoir un partage de la VA entre seuls salaires et profit, on aura un partage élargi entre rémunérations du travail, rétribution du capital et facteurs contribuant à long terme au développement des entreprises (voir Figure 2). L'amortissement pourrait ainsi être décomposé en trois sous-amortissements : du patrimoine-productif, du patrimoine humain et du patrimoine naturel.

Il s'agit de faire figurer au passif du bilan, en tant que dettes de l'entreprise, non plus le seul capital financier, mais également les coûts de maintien ou de conservation des êtres humains et de la nature. 

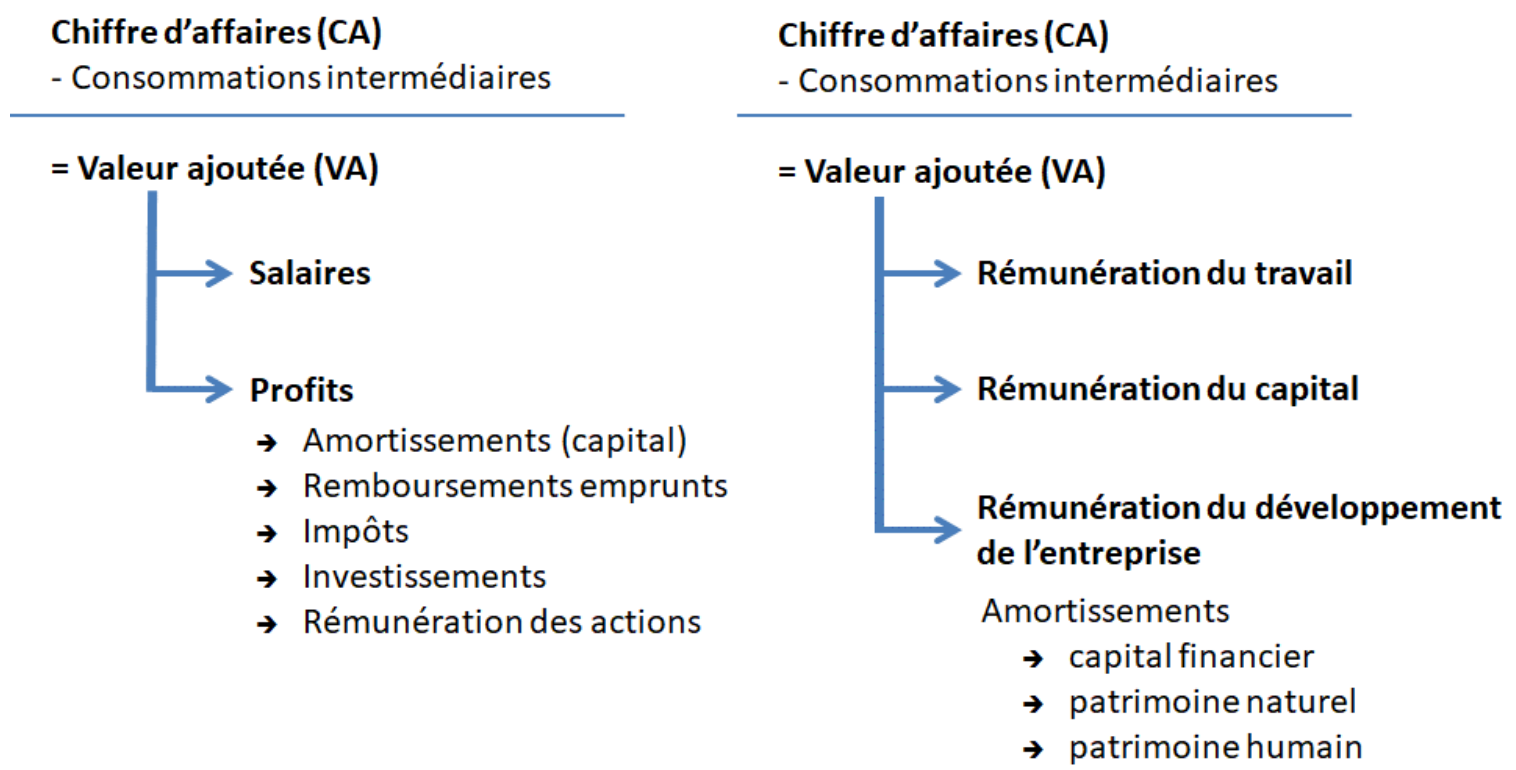

Figure 2. Normes comptables en vigueur et proposition de réforme de ces normes (à partir de [BAC13] et [BAC19])

Comme le souligne Daniel Bachet [BAC 13] [BAC 19], cette approche permettrait d'empêcher les atteintes aux fonctions environnementales essentielles et à la survie de la biosphère, et de prévenir les dégâts du développement économique sur les humains (risques socio-psychologiques, accidents, coût de l'insécurité environnementale, etc.). Cette nouvelle comptabilité serait le soubassement comptable d'une redéfinition radicale de l'entreprise. L'entreprise ne serait plus conçue comme un instrument de production et de profit mais une entité collective soucieuse des ressources naturelles et respectueuse du travail humain.

La logique serait de préserver le patrimoine environnemental et humain au même titre que le capital financier. La tenue de ce type de comptabilité serait de nature à inciter l'entreprise à organiser une veille, être en alerte et prévenir rapidement, par exemple, les dépassements de pollution. Il s'agirait de concevoir un profit qui ne dégrade pas les ressources naturelles, humaines et financières de l'entreprise.

Ce meilleur partage entre rémunérations du travail et du capital devrait déboucher sur une augmentation du salaire dans les (grandes) entreprises et dans certaines fonctions publiques comme l'hôpital. Cette hausse des moyens pour vivre est d'autant plus justifiée lorsque l'on regarde l'évolution de la productivité du travail (la quantité de produit et de service produite avec une heure de travail). Cela permet d'insister sur le partage inégalitaire au cours du temps de la valeur ajoutée, entre le travail et le capital. Une heure de travail permettait de produire 19 euros de marchandises ou de services en 1970 et 55 euros en $2015^{7}$.

\subsection{Les conventions de contextualisation}

Si l'on prend l'exemple de la dette publique, celle-ci est le plus souvent rapportée en \% du PIB. Le ratio est alors de $220 \%$ pour le Japon, de $185 \%$ pour la Grèce et de $100 \%$ pour la France. On est alors enclin de considérer, par échelle de "gravité », que la dette la plus inquiétante est la dette japonaise et que celle qui l'est le moins est celle de l'État français.

\footnotetext{
${ }^{7}$ Chiffres exprimés en parité de pouvoir d'achat et en euros de 2015 , c'est-à-dire après prise en compte de l'inflation et du niveau des prix dans les différents pays. Voir https://www.lemonde.fr/blog/piketty/2017/01/05/de-la-productivite-en-france-en-allemagne-etailleurs/ 
Mais la dette du Japon est presqu'exclusivement détenue par des résidents alors que la dette de la France et celle de la Grèce sont largement détenues par des non-résidents (respectivement, $60 \%$ et 100\%). Cette détention relativise, contextualise, la gravité de la dette.

La combinaison des deux données (le montant de la dette publique, d'une part, le PIB ou la part de la dette détenue par les non-résidents, d'autre part), qui produit une information, ne produit pas le même jugement sur la gravité de la dette publique selon le choix de combinaison de données effectué.

C'est ce choix que nous appelons « convention de contextualisation».

Proches des conventions de quantification, les conventions de contextualisation (qui elles-mêmes reposent sur des choix de quantification) ne se confondent pas avec ces premières. Elles suggèrent un sens, une interprétation particulière, un contexte. Leur caractère situé fait qu'elle assurent le passage de la donnée à l'information.

Bien connu des statisticiens, le paradoxe de Simpson permet d'illustrer comment la présentation des données offre la possibilité de modifier l'information que l'on souhaiter présenter. Nous renvoyons à la littérature [DEL 13] pour davantage d'explications sur la nature de ce paradoxe et nous nous contenterons ici de l'illustrer avec l'exemple fictif d'une entreprise dont les employés sont répartis en deux catégories (catégorie A et catégorie B) et dont la direction souhaite mettre en avant les efforts faits pour rattraper les écarts de rémunérations entre personnels femmes $(\mathrm{F})$ et hommes $(\mathrm{H})$, notoirement en défaveur des premières. La direction annonce ainsi que les augmentations de salaires et primes ont été cette année davantage favorables aux femmes qu'aux hommes, chiffres à l'appui : dans la catégorie A, $100 \%$ des femmes en ont bénéficié contre $84 \%$ des hommes tandis que dans la catégorie B, 56\% des femmes en ont bénéficié contre $40 \%$ des hommes.

Le Tableau 1 donne les valeurs précises confirmant ce qu'avance la direction.

\begin{tabular}{|c|c|c|c|c|c|c|}
\hline & \multicolumn{2}{|c|}{$\begin{array}{c}\text { Employés } \\
\text { catégorie A }\end{array}$} & \multicolumn{2}{|c|}{$\begin{array}{c}\text { Employés } \\
\text { catégorie B }\end{array}$} & \multicolumn{2}{|c|}{$\begin{array}{l}\text { Tous les } \\
\text { employés }\end{array}$} \\
\hline & $\mathbf{F}$ & $\mathbf{H}$ & $\mathbf{F}$ & $\mathbf{H}$ & $\mathbf{F}$ & $\mathbf{H}$ \\
\hline $\begin{array}{c}\text { Bénéficiaires } \\
\text { d'augmentations }\end{array}$ & 5 & 38 & 25 & 2 & 30 & 40 \\
\hline $\begin{array}{l}\text { Nombre total } \\
\text { d'employés }\end{array}$ & 5 & 45 & 45 & 5 & 50 & 50 \\
\hline $\begin{array}{c}\text { Pourcentage de } \\
\text { bénéficiaires }\end{array}$ & $100 \%$ & $84 \%$ & $56 \%$ & $40 \%$ & $60 \%$ & $80 \%$ \\
\hline
\end{tabular}

Tableau 1. Augmentations de salaires selon catégorie et sexe des employés

Pourtant, ce même tableau montre que, lorsqu'on considère les employés de l'entreprise indistinctement (c'est-à-dire, en mélangeant catégories A et B), les augmentations de salaires et primes ont en réalité été davantage favorables aux hommes qu'aux femmes puisque $80 \%$ des hommes en ont bénéficié contre $60 \%$ des femmes !

Il ne nous semble pas exagéré de penser que la convention de contextualisation adoptée ou imposée dépend de la vision que l'on a de l'objet contextualisé. Dans l'exemple de la dette, si l'acteur qui «manipule» les chiffres de la dette se réfère à la dette publique française, ou grecque a fortiori, et souhaite montrer que cette dette est très inquiétante, il préférera la situer en rapport à la part de la dette détenue par des non-résidents. 


\subsection{Les conventions d'énonciation}

La manière d'énoncer une question ou un problème a un effet déterminant sur la façon dont cette question ou ce problème vont être appréhendés. Énoncer d'une certaine manière induit fréquemment implicitement un mode de résolution, souvent considéré comme unique [PAR 08].

À titre d'illustration, nous prendrons deux énoncés. Le premier concerne le déficit de la balance commerciale d'un pays comme la France. Le second a trait au déficit du budget de l'État.

À propos de la balance commerciale, il est souvent énoncé des propositions qui se résument comme suit : «La réduction du déficit de la balance commerciale de la France impose un effort accru pour soutenir l'exportation ».

Aucune mention n'est ici faite des importations qui peuvent apparaître comme le résultat de la désindustrialisation...

S'agissant du déficit du budget de l'État, il est énoncé la proposition suivante : «Le déficit du budget de l'État pèse sur la croissance et il est impératif de le réduire. Pour cela, la baisse des dépenses publiques est un objectif indispensable ».

Rien n'est expliqué du lien entre déficit et croissance, qui, dans l'analyse de Keynes, est souvent considéré non comme négatif mais comme positif. Aucune proposition fiscale n'est avancée pour taxer davantage les bénéfices du capital ou les revenus des plus riches. Enfin, rien n'est dit du lien inverse, cette fois entre croissance de l'activité et déficit. Une activité plus forte génère une hausse des recettes fiscales et une baisse des dépenses publiques liées à la «gestion » de la crise (la réduction du nombre de chômeurs a pour conséquence une baisse des allocations chômage sans que les allocations individuelles ne soient diminuées). D'où une possible réduction du déficit.

Sur un plan proche mais un peu différent, l'utilisation de pourcentages dans un énoncé peut avoir un effet déformant, voire faussant la perception que peut avoir celui qui prend connaissance des chiffres qui lui sont communiqués. À titre d'exemple, dans un même énoncé, une hausse du pourcentage de $100 \%$ sur une population faible a une incidence nettement moins forte qu'une hausse de $10 \%$ sur une population nombreuse.

\subsection{Les conventions sémiotiques}

La distinction introduite par Benveniste [BEN 74] entre sémantique - signification d'un mot, d'un chiffre ou d'un ensemble de mots et de chiffres - et sémiotique, nous permet d'insister sur la sémiotique en tant que mode de signifiance de ce qui est propre au signe. La sémiotique est l'étude des signes dans toutes leurs formes et dans toutes leurs manifestations, et de leur signification. Pour Peirce le signe est «tout ce qui communique une notion définie d'un objet, de quelque façon que ce soit » [PEI 78]. Le signe est « un véhicule qui communique à l'esprit quelque chose de l'extérieur » [PEI 78]. Ce peut être par exemple une forme, une couleur, une image, une échelle, etc.

Dans un univers où le visuel prend de plus en plus de place, les signes choisis pour exprimer une proposition ou un simple message peuvent induire une représentation particulière qui est celle que l'émetteur de la proposition souhaite de facto suggérer. Le signe agit sur une chaîne d'interprétation. La notion de sens n'est donc pas réductible à celle du signifié par des mots ou des chiffres mais est le produit de l'articulation entre le signifié et le signe qui lui est associé. Comme langage particulier, aucun signe ne peut être considéré comme «naturel». À titre d'exemple, le signe véhiculé par une couleur n'a rien de naturel comme le montrent les couleurs bleue et rose qui, de nos jours, renvoient respectivement aux garçons et aux filles alors que pendant longtemps c'était l'inverse ${ }^{8}$. De même, la couleur du deuil ou celle du mariage n'est pas identique dans chaque culture.

\footnotetext{
${ }^{8}$ Sur ce sujet, on peut lire : https://fr.aleteia.org/2018/05/26/quand-le-bleu-etait-pour-les-filles-et-le-rose-pour-les-garcons/ 
Les conventions sémiotiques que nous introduisons, complètent l'ensemble des conventions (de quantification, de contextualisation, d'énonciation et donc sémiotiques) qui s'articulent et s'agencent pour produire de l'information, pour passer de la donnée à l'information.

Le fait de choisir le vert ou le rouge dans un tableau, de retenir une échelle logarithmique sur un graphique, ont des incidences sur l'interprétation qui est suggérée par l'image qui est produite. La sémiotique retenue suggère une certaine interprétation, positive ou négative, ou encore accentue la perception que le destinataire du message peut avoir d'un phénomène ou d'un processus. Ce type de convention pourra être mis en lumière dans l'exemple du tableau de bord d'un hôpital que nous allons maintenant développer.

\section{Le tableau de bord d'une organisation : l'exemple de l'hôpital}

L'un des usages les plus importants des SI est de justifier et faciliter la prise de décisions variées, qu'elles soient stratégiques ou opérationnelles. Ce processus est mis en œuvre au sein de systèmes d'aide à la décision (SAD). Les indicateurs construits dans les SI et mobilisés dans les SAD énoncent des règles de comportements et traduisent des conventions implicites, qui elles-mêmes sont le reflet de la doxa. Le tableau de bord construit par les organisations à l'aide des SI est le révélateur d'une certaine vision du monde : pourquoi le choix de ces données ? Quelles informations sont-elles censées traduire ? Quelles sont les questions que l'on pose ? Pourquoi ?

Les tableaux de bord permettent de synthétiser des données sur l'organisation. Ces tableaux doivent permettre et faciliter la prise de décision. La première convention - convention d'énonciation - très souvent sous-entendue est que ces tableaux de bord sont censés rendre inutile et superflu le débat et la construction de compromis parce que ce qui est exact (en termes de calculs) est présumé être juste et légitime. La réflexion sur les processus de construction des indicateurs est tenue pour inutile et superflue. Or « les chiffres n'apportent ni la neutralité, ni l'objectivité qu'on voudrait leur accorder, ils participent à des constructions mentales » [FIE 18], à des conventions.

Nous pouvons décomposer cette construction de tableau de bord :

- les données sont choisies (ici pour le SI d'un hôpital : nombre de séjours, nombre de patients, séjours par pôles de l'hôpital, évolution des recettes, etc.).

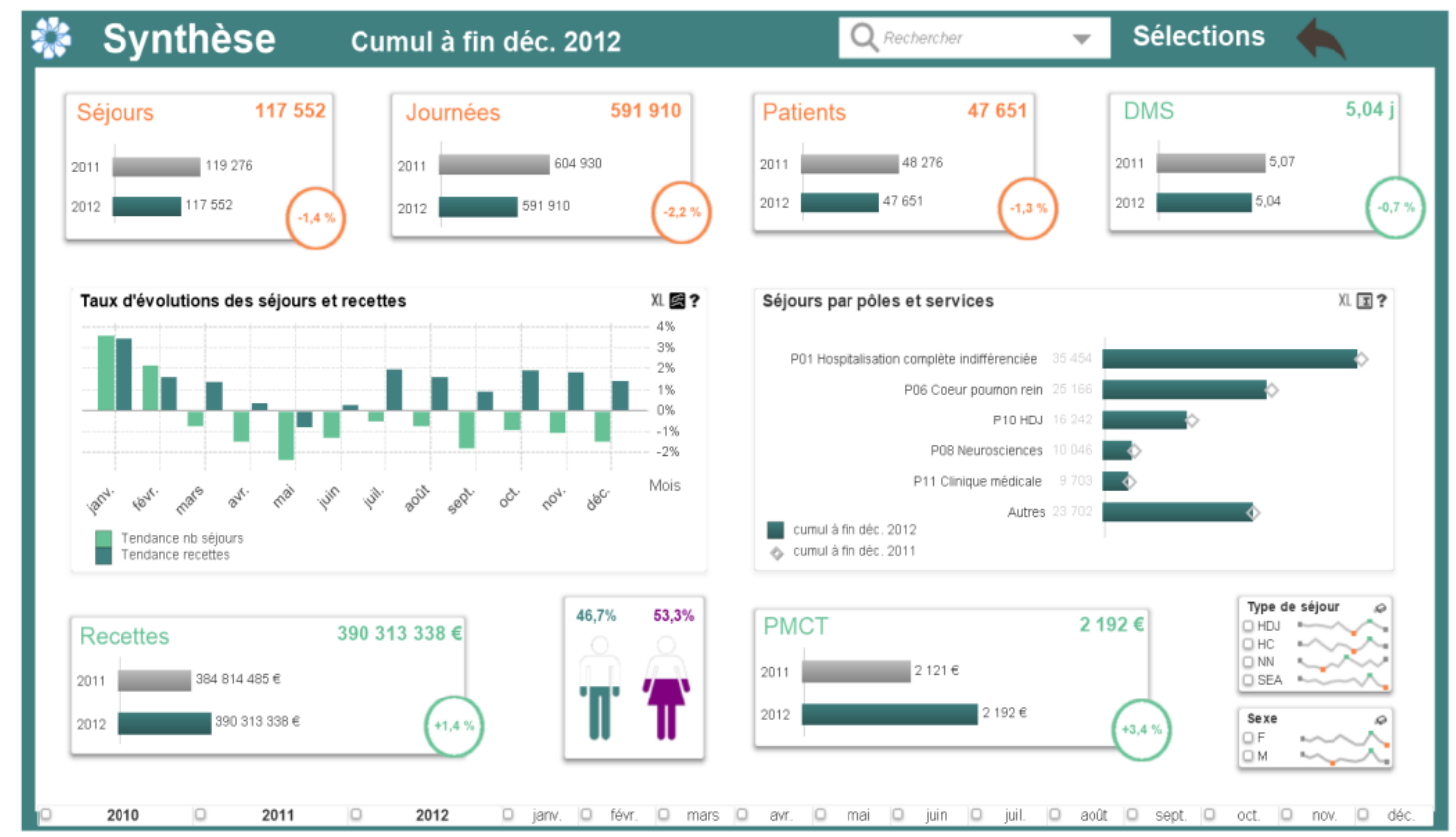

Figure 3. Tableau de bord d'un hôpital 
Il aurait été possible de capter d'autres données. Par exemple, puisque pour l'Office Mondial de la Santé, l'hôpital est une organisation qui doit assurer des soins médicaux complets, curatifs et préventifs, mais aussi une organisation qui assure l'enseignement de la médecine et de la recherche bio-sociale, on aurait pu aussi relever des données sur la qualité des soins, sur la formation du personnel, sur la recherche, etc. Les données choisies sont synthétisées au sein d'indicateurs. Il aurait été possible de construire d'autres indicateurs, de donner d'autres informations.

- les indicateurs sont combinés, corrélés de telle ou telle façon. Dans la figure ci-dessous par exemple, la corrélation suggérée de suivre est celle de l'évolution des séjours et celle des recettes.

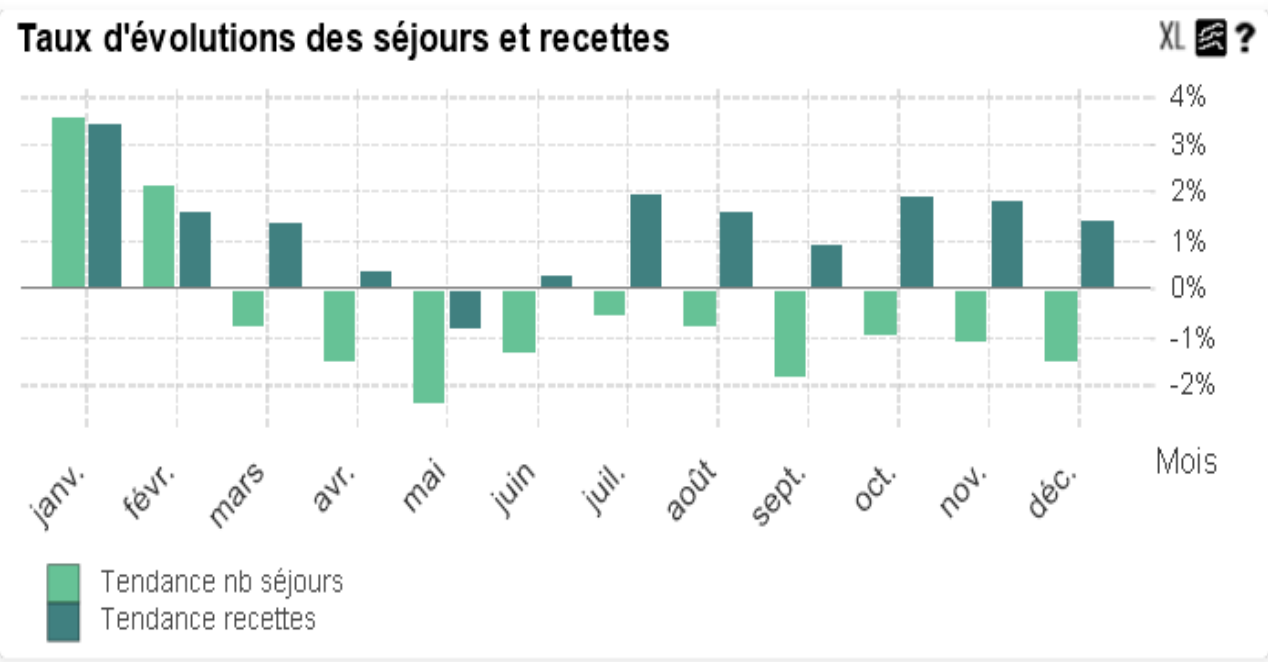

Figure 4. Graphique du taux d'évolution des séjours et recettes

On aurait pu construire d'autres corrélations en partant des mêmes données (évolution des séjours et évolution des pôles), et a fortiori, en partant d'autres données. Quelle est l'information qui est diffusée ? Dans cet exemple, on constate un renversement (heureux ?) de la tendance à partir de juin : les recettes ne varient plus dans le même sens que le nombre de séjours. Le nombre de séjours diminue alors que les recettes augmentent....

- à ces chiffres sont ajoutés des codes, souvent des codes de couleur supposés traduire l'objectif « naturel » de l'organisation. En vert, ce qui est bon pour l'organisation, en orange, ce qui est mauvais. La durée moyenne du séjour (DMS) diminue. Elle est en vert, ce qui suggère qu'il s'agit d'une valeur positive. Une première convention implicite apparaît ici, dès la construction du tableau de bord : il existe un objectif «naturel » de l'hôpital, diminuer la DMS.
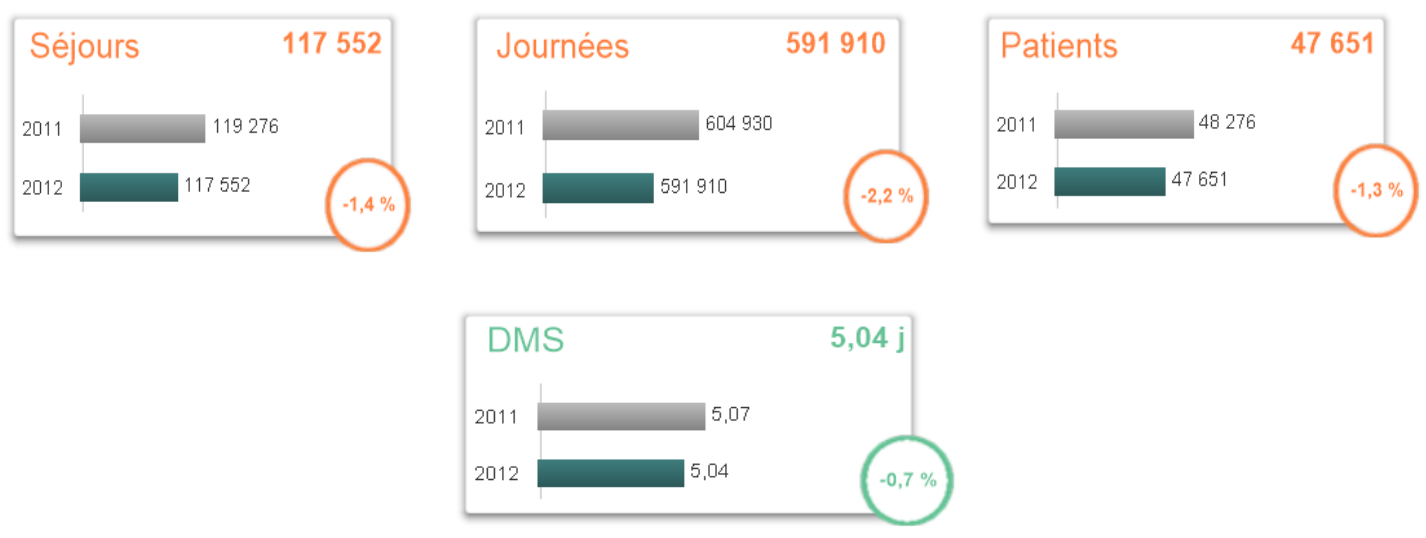

Figure 5. Évolution des nombres de séjours, journées, patients, et de la DMS 
Deuxième sous-entendu : le tableau de bord permet de faire apparaitre « objectivement » le but de l'organisation. La quantification ne change pas la nature de l'objectif de l'organisation, elle révèle ce dernier. La quantification est neutre axiologiquement ${ }^{9}$. C'est ce que les conventionnalistes qualifient de «convention industrielle»: gouvernement par les normes, standards, objectifs [ISL 17]. Naturellement, automatiquement, va apparaître l'objectif de l'organisation (en vert).

Troisième convention implicite : tout ce qui est fait plus rapidement est positif. La vitesse est une valeur positive en soi : idée reçue qui n'est jamais discutée. Ici, la durée moyenne du séjour. Pourtant, cette rapidité pourrait être discutée car elle peut recouvrir plusieurs réalités qui ne sont pas toutes forcément positives : la durée des séjours diminue parce que les patients sont mieux soignés, parce que la prise en charge à la sortie s'améliore, parce que les patients sont rendus plus autonomes, ou, dans des termes très différents, voire opposés, les séjours diminuent parce que les patients sont renvoyés chez eux sans être complétement guéris, parce qu'ils souhaitent partir plus vite car mal pris en considération lors de leur séjour, etc.

Dans l'exemple traité, on constate que la baisse du nombre de séjours, de journées, de patients, est considérée comme un processus négatif : la couleur rouge est employée. "Heureusement », ces baisses sont compensées par la baisse du ratio «durée moyenne de séjour » qui est considérée comme processus positif (marqué par la couleur verte). La corrélation établie entre le taux d'évolution des séjours et les recettes confirme cette tendance. Les recettes progressent d'une année sur l'autre alors que le nombre de séjours, de journées et de patients a diminué ... l'hôpital, la clinique, est plus rentable ! La rentabilité de l'organisation augmente, ce qui est positif. C'est la conclusion du tableau de bord. Les données ont été choisies, synthétisées et combinées pour aboutir à cet indicateur. Outre cette naïveté (ou contre-sens) de penser l'axiomatisation comme étant neutre [FIE 18], la convention marchande est placée en position supérieure et acquiert en même temps une extériorité qui la soustrait au débat; elle est posée comme une incontournable procédure d'agrégation [THE 10] [THE 06].

Il existe donc des conventions de quantification : que choisit-on de mesurer et comment le mesure-ton ? Quel est le nombre de séjours ? Il aurait été aussi possible de s'intéresser aux rechutes après la sortie de l'hôpital par exemple, ou à la formation continue des soignants qui pourraient aussi traduire une meilleure qualité des soins, ou le nombre de réunions collectives sur l'éthique...

Il existe aussi des conventions de contextualisation : quel est le contexte dans lequel on insère la mesure ? Doit-on comparer le nombre de séjours aux recettes de l'hôpital ou à un autre critère ?

Aux conventions de contextualisation, nous pouvons ajouter des conventions d'énonciation. Comment énonce-t-on ce que l'on envisage de mesurer ? La rentabilité d'un hôpital est prioritaire, un hôpital doit être rentable. La rentabilité de l'hôpital est énoncée sous une forme problématique pour induire le fait qu'il « faut résoudre ou affronter le problème ».

Il existe enfin des conventions sémiotiques : quels sont les signes utilisés pour produire telle ou telle image de la mesure ? La DMS (durée moyenne des séjours) baisse, le chiffre est donné en vert, il doit être considéré comme positif. Le débat sur l'aspect positif ou non de cet indicatif est ainsi clos a priori.

\section{Conclusion : les conventions sont trop rarement des compromis élaborés démocratiquement}

En même temps cadre et résultat de l'action, instituantes et instituées comme l'ont montré les « conventionnalistes » [DUP 89], les conventions et la dispute autour de celles-ci constituent le reflet de la vie de la démocratie dans les organisations. Lorsque les conventions sont implicites ou tacites, elles peuvent être le résultat d'une dispute qui n'a pas eu lieu. Elles sont alors imposées, de fait, par un acteur dominant, voire hégémonique, qui, ne les désignant pas, fait de celles-ci des normes allant de

\footnotetext{
${ }^{9} \mathrm{~S}$ 'opposant à cet axiome, Michael Sandel a voulu déconstruire l'hypothèse de la théorie économique dominante selon laquelle le marché est neutre. Selon cette théorie, "marchandiser", donner un prix à une chose ne modifie pas celle-ci [SAN 14]. 
soi. Les données sont alors présentées comme données, les informations comme exprimant des faits, non comme impliquant des jugements, des interprétations, des valeurs.

Au lieu de laisser la place aux tensions, aux dénonciations d'une convention légitime au nom d'une autre, la construction d'indicateurs lisse le différend en rendant invisible les conventions sous-jacentes. L'organisation est supposée plus performante et efficace si le différend est lissé. On suppose que le débat n'apporte pas un «plus» mais au contraire conduit à une perte de temps. Les différentes caractéristiques de la finalité de l'organisation ainsi que la discussion (démocratique) sur ces caractéristiques sont rejetées. Quelle est la finalité de l'hôpital : la rentabilité ou l'amélioration de la prise en charge de la vulnérabilité et de la dignité d'un patient, c'est-à-dire l'éthique ${ }^{10}$; ou les deux, et dans ce dernier cas, quelle est la hiérarchie entre ces objectifs ?

Comme le fonctionnement du SI en général et du SI numérique en particulier, on peut penser que la question de l'élaboration des conventions est trop souvent ignorée et son importance sous-estimée par les acteurs potentiels de la dispute, - syndicats ou représentants des personnels, par exemple -, dans les organisations.

S'agissant des syndicats, comme composante de la démocratie salariale, ceux-ci, largement affaiblis dans la période récente comme la plupart des institutions d'intermédiation, ne pourront à l'avenir pleinement jouer leur rôle et contester la gouvernance actionnariale ou managériale que s'ils investissent la conception et le fonctionnement des SI et s'engagent plus activement dans la production des conventions. Il serait alors possible d'imaginer que l'on retrouve dans cette démarche d'implication certains des éléments du projet d'« industrial democracy » des années 1960-1970 fort en vogue dans les pays scandinaves ou encore des questionnements présents dans le programme du Conseil national de la Résistance lorsque ce programme signalait comme un enjeu important la question de l'expertise des organisations syndicales.

\section{Bibliographie}

[BAC 13] BACHET D., COMPIN F. « De la finance au travail : finalités institutionnelles des entreprises et critères de gestion », La Pensée, n 375, p. 83-96, 2013.

[BAC 19] BACHET D., «Voir autrement l'entreprise pour émanciper le travail », Marianne, tribune publiée le 07/06/2019. En ligne : https://www.marianne.net/debattons/tribunes/voir-autrement-l-entreprise-pour-emanciper-letravail (consulté le 30 mars 2020).

[BAT 79] BATESON G., Mind and Nature: A Necessary Unity, Hampton Press, New York, 1979.

[BEN 74] BENVENISTE E., Problèmes de linguistique générale, Tome II, Gallimard, Paris, 1974.

[BER 83] BERRY M., Une technologie invisible? L'impact des instruments de gestion sur l'évolution des systèmes humains, Centre de recherche en gestion (CRG) de l'École Polytechnique, Paris, 1983.

[DEL 13] Delahaye J.-P., « L’embarrassant paradoxe de Simpson », Pour la Science, n429, p. 80-85, 2013.

[COL 19] Colletis G., FieuX E., ISla A., Peneranda A., «De la donnée à l'information : l'importance des conventions », dans Ateliers INFORSID, Université Paris Dauphine, Paris, 11/06/2019-14/06/2019, 2019. En ligne : https://si2d.hypotheses.org/1805 (consulté le 30 mars 2020).

[DES 03] DESROSIERES A., " Naissance d'un nouveau langage statistique entre 1940 et 1960 », Courrier des Statistiques, $\mathrm{n}^{\circ} 108$, p. 41-52, décembre 2003.

[DES 10] DESROSIERES A., La politique des grands nombres. Histoire de la raison statistique, La Découverte, Paris, 2010 .

[DUP 89] DUPUY J.P., EYMARD-DUVERnAY F., FAVEREAU O., OrlEAN A., SAlais R., ThEVENOT L., « Introduction », Revue économique, vol. 40, n², «L’économie des conventions », p 141-145, 1989.

${ }^{10}$ Sur l'éthique à l'hôpital, voir [HAR 07]. 
[EYM 06] Eymard-Duvernay F., FAVEReau O., ORlean A., SAlais R., TheVEnOt L., « Des contrats incitatifs aux conventions légitimes. Une alternative aux politiques néo-libérales », dans EYMARD-DUVERNAY F. (ed.), L'économie des conventions, méthodes et résultats, T.II Développements, éditions La Découverte, collection Recherches, Paris, pp.17-42, 2006.

[FAV 89] FAVEREAU O., « Marchés internes, marchés externes », Revue Economique, vol. 40(2), p. 273-328, 1989.

[FIE 18] FIEUX E. (2018). "Quantifier, c'est déjà décider », dans Ateliers INFORSID, Nantes, 28/05/2018-31/05/2018, 2018. En ligne : https://si2d.hypotheses.org/1024 (consulté le 30 mars 2020).

[FRI 92] FRIEDBERG E., « Les quatre dimensions de l'action organisée », Revue française de sociologie, $\mathrm{n}^{\circ}$ XXXIII-4, p. 531-557, octobre-décembre 1992.

[HAR 07] HARDY L., «L'hôpital en marche vers une éthique de tous les métiers ? », Recherche en soins infirmiers, 2007/3 (N 90), p. 95-101, 2007.

[ISL 02] ISLA A., «Pour une modélisation économique complexe de la prise de décision juridique : quelques pistes de recherche », Cahiers du Lereps, n²002-1.

[ISL 05] ISLA A., L'économie comme "science humaine", dossier pour l'obtention de l'Habilitation à Diriger des Recherches, Université Toulouse-1, 2005.

[ISL 17] ISLA A., «Évolution des conventions de qualité et enjeux de la singularité sur le marché du vin », Systèmes alimentaires/Food Systems, n², p. 197-217, 2017.

[ISL 19] ISLA A., «La légitimité des revendications économiques des Gilets Jaunes », Conférence faite à la Bourse du Travail de Tarbes, le 6 novembre, à l'initiative du collectif des Gilets Jaunes de Tarbes (CSGJ65), 2019. En ligne : https://hal.archives-ouvertes.fr/hal-02355838 (consulté le 30 mars 2020).

[LEM 86] LE Moigne J.-L., «Vers un système d'information organisationnel ?», Revue Française de Gestion, novembre-décembre, 1986.

[KIR 02] KiRat T., Raveaud G., «BATIFoulier Ph. (dir.) Théorie des conventions, Economica, Paris, septembre 2001 », Économies et institutions, $\mathrm{n}^{\circ} 1 / 2002$. En ligne : https://journals.openedition.org/ei/714 (consulté le 31 mars 2020).

[LEM 90] Le MoIGNE J.-L., La modélisation des systèmes complexes, Dunod, Bordas, Paris, 1990.

[LEM 95] Le Moigne J.-L., «Sur la Modélisation Systémique de l'Information », Séminaire «Économie de l'Information », Commissariat Général du Plan, Paris, 19 septembre 1995.

[LEM 06] LE Moigne J-L., AMABILE S., « Épistémologie des systèmes d'information », dans I. WATTIAU I. et J. AKOKA, (ed.), Encyclopédie des Systèmes d'Information et de l'informatique, Vuibert, Paris, 2006.

[LYM 03] LyMAN P., VARIAN H. R., « How Much Information? », School of Information Management and Systems, University of California, Berkeley, 2003.

[MAR 64] MARCH J., SiMON H., Organisations, Economica, Paris, 1964.

[MEL 79] MELESE J., Approches systémiques des organisations. Vers l'entreprise à complexité humaine, Hommes et Techniques, Paris, 1979.

[MOR 77] MoRin E., La méthode, Tome 1 : La Nature de la Nature, Tome 2 : La Vie de la Vie, éditions du Seuil, collection Point anthropologie, Sciences humaines, Paris, 1977.

[NOD 11] Node-LangloIs M., Disputes philosophiques, éditions Artège, Paris, 2011.

[PAR 08] PARADICE D., "Decision Support and Problem Formulation Activity », Encyclopedia of Decision Making and Decision Support Technologies, IGI Global, Hershey, PA, 2008.

[PEI 78] PEIRCE C.-S., Écrits sur le signe, Edition du Seuil, collection L'ordre philosophique, Paris, 1978.

[RAM 15] RAMBAUD A. P., La valeur de l'existence en comptabilité : pourquoi et comment l'entreprise peut prendre en compte des entités environnementales pour «elles-mêmes »?, Thèse de doctorat en Sciences de gestion, Paris Dauphine, 2015.

[RIC 19] RICHARD J., «Changer de comptabilité pour sauver la planète», En ligne : https://www.liberation.fr/debats/2019/09/16/changer-de-compta-pour-sauver-la-planete_1751610 (consulté le 31 mars 2020).

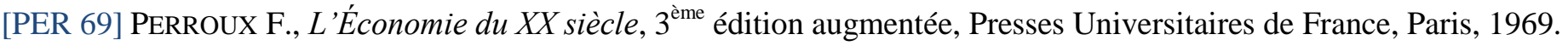


[SAL 10] SAlais R., «La donnée n'est pas un donné. Pour une analyse critique de l'évaluation chiffrée de la performance », Revue française d'administration publique, $\mathrm{n}^{\circ}$ 2010/3 (n 135), p. 497-515, 2010.

[SAL 13] SAlles M., Colletis G., « Déconstruire la doxa dominante, construire une pensée politique alternative. Du lien entre les représentations, les principes et les normes », Lo Sguardo, "The Instruments of the Power», XIII, 10/2013, p. 391-414, 2013.

[SAL 15] SAlles M., Décision et système d'information, Collection Systèmes d'information avancés, Vol. 2, ISTEWILEY, Londres, 2015.

[SAL 20] SALLES M., BOUR R., JARDAT R. «Systèmes d'information numériques : supports ou entraves à la démocratie dans les organisations ? », Revue Ouverte de l'Ingénierie des Systèmes d'Information, Volume 20-1, n² 2020.

[SAN 14] SANDEL M., What Money Can't Buy, Macmillan, New York, 2012. (Traduction française : Ce que l'argent ne saurait acheter, Éditions du Seuil, Paris, 2014).

[SAU 14] SAUVAJOL-Rialland C., «Infobésité, gros risques et vrais remèdes », L'Expansion Management Review, 2014/1, n 152, p. 110-118, 2014.

[SIM 80] SimON H., Le nouveau management : la décision par les ordinateurs, Economica, Paris, 1980.

[SIM 83] Simon H., Administrative Behavior. A Study of Decision-Making Processes in Administrative Organization, Third Edition, The Free Press, Collier Macmillan Publishers, London, UK, 1976. (Traduction française : Administration et processus de decision, Economica, Paris, 1983).

[SIM 91] SIMON H., «Bounded Rationality and Organizational Learning », Organization Science, 2 (1), p. 125-134, 1991.

[THE 06] THEVENOT L., L'action au pluriel, sociologie des régimes d'engagement, Éditions La Découverte, Paris, 2006.

[THE 10] THÉvENOT L., « Autorità e poteri alla prova della critica. L'oppressione del governo orientato all 'obiettivo' », Rassegna Italiana di Sociologia, n 2010. 